\title{
ESTUDIO RETROSPECTIVO SOBRE LA INCIDENCIA Y TRATAMIENTO DE EPISTAXIS EN UN ÁREA DE SALUD EN EL PERÍODO 2011 A 2017
}

\section{Retrospective study about the incidence and treatment of epistaxis in a health area in the period 2011 to 2017}

\author{
Elena HELLÍN-VALIENTE; Esteban MERINO-GÁLVEZ; Diego HELLÍN-MESEGUER \\ Hospital Rafael Méndez. Servicio de Otorrinolaringología. Lorca. Murcia. España. Hospital Clínico Universitario \\ Virgen de la Arrixaca. Murcia. España \\ Correspondencia: elena_07_02@hotmail.com
}

Fecha de recepción: 13 de junio de 2018

Fecha de aceptación: 12 de julio de 2018

Fecha de publicación: 13 de julio de 2018

Fecha de publicación del fascículo: 15 de marzo de 2019

Conflicto de intereses: Los autores declaran no tener conflictos de intereses

Imágenes: Los autores declaran haber obtenido las imágenes con el permiso de los pacientes

Política de derechos y autoarchivo: se permite el autoarchivo de la versión post-print (SHERPA/RoMEO)

Licencia CC BY-NC-ND. Licencia Creative Commons Atribución-NoComercial-SinDerivar 4.0 Internacional

Universidad de Salamanca. Su comercialización está sujeta al permiso del editor

\begin{abstract}
RESUMEN: Introducción y objetivo: La epistaxis es un signo clínico frecuente y un motivo de consulta urgente habitual. Tenemos escasos datos sobre su epidemiología y el tratamiento aplicado en nuestra Región. Los objetivos del estudio son conocer su incidencia en nuestro medio, conocer sus características epidemiológicas, determinar factores asociados a ella y conocer sus distintos tratamientos. Método: Estudio observacional retrospectivo que describe y analiza los pacientes atendidos por epistaxis en la Unidad de Urgencias del Hospital Rafael Méndez, de Lorca (Murcia, España), durante un período de 7 años. Se estudian 2.138 pacientes. Resultados: La incidencia global fue de 1,75 casos /1.000 habitantes por año. El 96,5\% fueron anteriores. Predominio en varones, ratio hombre/mujer de 2/1. Fue más frecuente en mayores de 50 años, con una edad media de 54,94 años. La mayor incidencia se dio en primavera e invierno. El 96\% del total fueron dados de alta a domicilio. Presenta relación con la hipertensión arterial, aunque sin significación estadística. El tratamiento más utilizado en general fue taponamiento anterior. El $46 \%$ de las epistaxis posteriores precisó tratamiento quirúrgico, con una estancia media hospitalaria de 6 días. Conclusiones: Las epistaxis atendidas en urgencias suelen tener un origen anterior, afectar a varones mayores de 50 años
\end{abstract}




\section{ESTUDIO RETROSPECTIVO SOBRE LA INCIDENCIA Y TRATAMIENTO DE EPISTAXIS \\ EN UN ÁREA DE SALUD EN EL PERÍODO 2011 A 2017 \\ HELLÍN-VALIENTE E ET AL}

con comorbilidad añadida, y suelen resolverse de manera ambulatoria. Las epistaxis posteriores requieren ingreso hospitalario y, con frecuencia, tratamiento quirúrgico.

PALABRAS CLAVE: Epistaxis; urgencias ORL; sangrado; epidemiología; hipertensión; tratamiento.

SUMMARY: Introduction and objective: Epistaxis is a frequent clinical sign and it is a usual reason for urgent consultation. We have limited data about its epidemiology and its treatment in our Region. The objectives of the study consist on: to know its incidence in our area and its epidemiological characteristics, to identify the factors associated with it and to know its different treatments. Method: An observational retrospective study that describes and analyzes all patients treated for epistaxis in the emergency department in Rafael Méndez Hospital, in Lorca (Murcia, Spain), during a period of 7 years. We studied 2.138 patients. Results: The global incidence was 1.75 cases /1,000 inhabitants per year. $96.5 \%$ were anterior epistaxis. Predominance in males, male: female ratio of $2: 1$. More frequent in people over 50 years old, with an average age of 54.94 years. The highest incidence was in spring and winter. $96 \%$ of the total were discharged from hospital. It is related to arterial hypertension, although without statistical significance. The most commonly used treatment was anterior nasal packing. $46 \%$ of the posterior epistaxis required surgical treatment, with a mean hospital stay of 6 days. Conclusions: Epistaxis seen in the emergency department usually has a anterior origin, affects men older than 50 years with added comorbidity, and usually resolves on an outpatient basis. Posterior epistaxis requires hospital admission and, frequently, surgical treatment.

KEYWORDS: Epistaxis; ENT emergencies; bleeding; epidemiology; hypertension; treatment.

\section{INTRODUCCIÓN}

Con el término epistaxis se define toda hemorragia con origen en las fosas nasales. Esta palabra deriva del griego y significa fluir gota a gota [1]. La localización anatómica de la nariz como apéndice que sobresale en el centro de la cara, sujeta a todo tipo de traumatismos. La abundante vascularización de las fosas nasales hace que este signo se presente con gran frecuencia. Aunque es un problema muy común, los datos sobre la incidencia y prevalencia de la epistaxis son muy variables, y probablemente infravalorados, ya que la mayoría de los episodios son autolimitados y no precisan atención médica. Globalmente se estima que el $60 \%$ de la población puede presentar al menos un episodio de epistaxis a lo largo de su vida, pero solo entre un 6-10\% de ellos necesitará asistencia médica [2-5]. En la mayoría de los casos, se produce en escasa cuantía y se resuelve de forma espontánea, pero en ocasiones, estos episodios pueden alcanzar mayor gravedad, poniendo en riesgo, incluso, la vida del paciente
[6]. Las epistaxis anteriores son las más frecuentes (90\%) [5, 7, 8-11], y la mayoría de ellas ceden de forma espontánea o con tratamiento conservador realizado por el propio paciente, sin necesidad de asistencia médica [12]. Hasta el $90 \%$ de estos sangrados se originan en un área hipervascularizada en el septo nasal, Plexo de Kiesselbach o Área de Little [13], lugar donde se anastomosan ramas de las arterias esfenopalatina, etmoidal anterior y labial. Se producen generalmente en jóvenes y niños, y suelen ser de buen pronóstico [14]. Las epistaxis posteriores son menos frecuentes (10\%), $y$ tienen su origen en el plexo de Woodruff, en las ramas posteriores de la arteria esfenopalatina, rama de la carótida externa y también puede originarse de la arteria etmoidal posterior, rama de la arteria carótida interna [13]. Se suelen presentar principalmente en adultos, tienen una resolución complicada y el pronóstico puede llegar a ser grave. En ellas es difícil encontrar el lugar de origen del sangrado [14]. Aunque la mayoría de las epistaxis se consideran esenciales o idiopáticas, al no conocer la causa 


\section{ESTUDIO RETROSPECTIVO SOBRE LA INCIDENCIA Y TRATAMIENTO DE EPISTAXIS \\ EN UN ÁREA DE SALUD EN EL PERÍODO 2011 A 2017 \\ HELLÍN-VALIENTE E ET AL}

[14], en ocasiones sí se identifica la misma, bien sea de causa local (traumatismo, cuerpo extraño, úlcera...) o de causa sistémica (medicamentos, alteraciones vasculares). Para realizar un manejo adecuado de la hemorragia nasal se debe tratar la epistaxis como un síntoma, el cual se debe aliviar, es decir detener el sangrado en primera instancia para luego identificar la causa. En la actualidad no existe un tratamiento ideal para la epistaxis, existe controversia sobre cuál es el tratamiento más adecuado como primer acto médico, si la cauterización o el taponamiento [12]. De lo que no cabe duda es que la epistaxis es un proceso muy frecuente que una buena parte de la población ha experimentado o experimentará alguna vez en su vida, aunque, debido a que la mayoría no requieren atención médica, su incidencia y prevalencia son difíciles de calcular. Aun así, supone una de las urgencias más frecuentes de la especialidad [15].

No tenemos datos reales sobre la incidencia en nuestra región y, por ello, planteamos este estudio, con el objetivo principal de conocer la incidencia y prevalencia de epistaxis en el Área III de Salud de Murcia (España), además de conocer las principales características epidemiológicas de los pacientes ingresados por epistaxis, determinar algunos posibles factores causales y conocer sus distintos tratamientos.

\section{MATERIAL Y MÉTODO}

Realizamos un estudio observacional retrospectivo de los pacientes atendidos en el servicio de Urgencias del Hospital Rafael Méndez de Lorca, entre los años 2011 y 2017. Recogimos los datos del total de pacientes atendidos en urgencias de nuestro hospital y periodo de tiempo indicado (536.287 pacientes). De ellos, se extrajo los datos de aquellos que fueron de causa de otorrinolaringología (ORL) (57.439 pacientes). Finalmente, se separaron aquellos que acudieron por epistaxis (2.138 pacientes). En ellos se describieron y analizaron las variables estudiadas en ambos grupos, epistaxis anterior
(2.064 pacientes) y epistaxis posterior (aquellas epistaxis en la que no se objetivó un punto de sangrado anterior activo o sospechoso) (74 pacientes). Por último, se obtuvo una muestra aleatoria, para la cual se consultaron las historias clínicas de 90 pacientes con epistaxis anterior y 50 pacientes con epistaxis posterior, para estudiar otras variables más específicas acerca de los antecedentes de los pacientes y el tratamiento realizado, y comparamos así las variables estudiadas entre ambos grupos de la muestra. En el caso de las epistaxis anteriores se aleatorizó según el número de historia clínica (NHC), En el caso de las epistaxis posteriores, se consultaron los informes de alta de los ingresados. Se solicitó permiso al Comité de Investigación del Área III de Salud de la Región de Murcia, a la que pertenece el hospital Rafael Méndez. Las variables analizadas se encuentran en las tablas 1, 2 y 3 . Se realizó análisis estadístico mediante el software estadístico SPSS 22.0 (IBM, Armonk, NY).

\section{RESULTADOS}

La población de referencia censada del área sanitaria del Hospital Rafael Méndez en el año 2017 era de 174.416 habitantes.

Durante el período estudiado acudieron 536.287 pacientes a urgencias, de los cuales 57.439 $(10,71 \%)$ fueron por patología ORL. De ellos, 2.138 pacientes consultaron por epistaxis. Esto representaba el $3,72 \%$ de todas las urgencias ORL atendidas en general en urgencias, y el 10,32\% de las urgencias atendidas por médicos especialistas ORL. El motivo de consulta ORL más frecuente en la puerta de urgencias fue catarro o infección vías altas (19,7\% de las urgencias ORL). Con la tasa de frecuentación estudiada, las epistaxis totales supusieron una incidencia de 1,754 casos por 1.000 habitantes y año. De ellas, las epistaxis anteriores representaban 1,685 casos por 1.000 habitantes y año, $y$ las posteriores 0,063 casos por 1.000 habitantes y año calculado en base a la población atendida por nuestro centro sanitario que es de 174.416 habitantes. 


\section{ESTUDIO RETROSPECTIVO SOBRE LA INCIDENCIA Y TRATAMIENTO DE EPISTAXIS EN UN ÁREA DE SALUD EN EL PERÍODO 2011 A 2017 \\ HELLÍN-VALIENTE E ET AL}

Tabla 1. Datos epistaxis general.

\begin{tabular}{|c|c|c|c|}
\hline & & $\begin{array}{l}\text { Número } \\
\text { pacientes }\end{array}$ & $\begin{array}{l}\text { Frecuencia } \\
\text { respecto al } \\
\text { total }\end{array}$ \\
\hline \multirow{2}{*}{ Tipos } & Anterior & 2.064 & $96,50 \%$ \\
\hline & Posterior & 74 & $3,50 \%$ \\
\hline \multirow{2}{*}{ Sexo } & Hombre & 1.384 & $64,70 \%$ \\
\hline & Mujer & 754 & $35,30 \%$ \\
\hline \multirow{4}{*}{ Edad } & $<15$ años & 248 & $11,60 \%$ \\
\hline & 15 - 21 años & 109 & $5,10 \%$ \\
\hline & 22 - 50 años & 386 & $18,10 \%$ \\
\hline & $>50$ años & 1395 & $65,20 \%$ \\
\hline \multirow{5}{*}{$\begin{array}{l}\text { Situación } \\
\text { al alta }\end{array}$} & Domicilio & 2.056 & $96,20 \%$ \\
\hline & Hospitalización & 64 & $3 \%$ \\
\hline & Traslado & 6 & $0,3 \%$ \\
\hline & Alta voluntaria & 1 & $0 \%$ \\
\hline & Fuga & 11 & $0,5 \%$ \\
\hline \multirow{3}{*}{$\begin{array}{l}\text { Unidad } \\
\text { de } \\
\text { Gestión }\end{array}$} & Urgencias & 1.166 & $54,50 \%$ \\
\hline & ORL & 949 & $44,40 \%$ \\
\hline & Pediatría & 23 & $1,10 \%$ \\
\hline
\end{tabular}

Del total de urgencias atendidas por epistaxis, 1.384 eran varones $(64,7 \%)$ y 754 mujeres $(35,3 \%)$ En cuanto a la localización de la epistaxis, el $96,5 \%$ fueron anteriores y el $3,5 \%$ restantes fueron de origen posterior.

Respecto a la edad, se dividió a la población en grupos etarios que nos parecieron más homogéneos para lograr mayor significación en el contraste, donde el 11,6\% fueron menores de 15 años, 5,1\% tenían entre 15 y 21 años, el 18,1\% tenían entre 22 y 50 años y el $65,2 \%$ restante tenían más de 50 años (1.395 pacientes). La edad media fue de 54,94 años, con una desviación típica de 25,429.

Del total de pacientes atendidos por epistaxis, solo 64 pacientes $(3 \%)$ precisaron hospitalización en nuestro centro mientras que 9 fueron derivados para embolización a nuestro hospital de referencia, siendo el resto alta domiciliaria tras la primera atención.

La atención médica fue prestada por especialistas ORL en el 44,4\% de los pacientes (949), y el resto por médicos de urgencias, salvo el $1 \%$ que fue atendido por pediatras (23 pacientes) La mayoría de los pacientes pertenecían a la Región de Murcia (98\%), correspondiendo todos ellos al Servicio Murciano de Salud. El 58\% de ellos residían en Lorca y el 14\% en Totana-WAledo.

a tasa de frecuentación anual por epistaxis en los años estudiados oscilaba entre 260 (en el año 2011) y 345 pacientes (en el año 2014).

Los meses con mayor frecuencia de epistaxis atendidas fueron enero, marzo, abril y mayo, oscilando entre el 10,4\% a 11,2\% (223 a 240 pacientes). Los meses de menor frecuencia fueron agosto, septiembre y octubre, oscilando entre $5,1 \%$ a $5,6 \%$ (110 a 120 pacientes). Esta variación estacional se muestra en Figura 1.

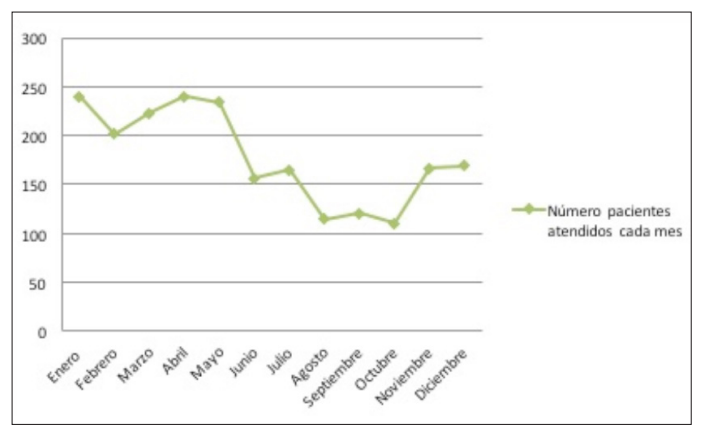

Figura 1. Variación estacional.

El día de la semana de mayor atención fue lunes, el 18,3\% de las epistaxis totales y el de menor atención fue domingo $(8,9 \%)$

Cuando comparamos las epistaxis anteriores y posteriores con las variables epidemiológicas descritas (Tabla 2), observamos que ambos tipos de epistaxis fueron más frecuentes en mayores de 50 años. Las epistaxis posteriores fueron poco frecuentes en menores de 15 años (4\%), siendo significativo comparándolas con las anteriores que representaban el $11,8 \%(\mathrm{p}<0,05)$. En cuanto al sexo, ambos tipos de epistaxis fueron más frecuentes en varones $(p>0,1)$, sin llegar a ser significativa. 


\section{ESTUDIO RETROSPECTIVO SOBRE LA INCIDENCIA Y TRATAMIENTO DE EPISTAXIS EN UN ÁREA DE SALUD EN EL PERÍODO 2011 A 2017 \\ HELLÍN-VALIENTE E ET AL}

Respecto a la situación al alta, la mayoría de epistaxis anteriores $(99,4 \%)$ fueron dadas de alta a domicilio, mientras que la mayoría de epistaxis posteriores $(86,48 \%)$ precisaron hospitalización y 6 pacientes traslado a otro hospital. Se demostró una diferencia estadística muy significativa $(\mathrm{p}<0,001)$.

La mayoría de las epistaxis posteriores fueron atendidas por médicos especialistas ORL (63,5\%), a diferencia de las anteriores que fueron atendidas por médicos de urgencias en el $55,23 \%$ y el $43,70 \%$ por especialistas ORL $(\mathrm{p}<0,05)$.

Al comparar ambos grupos de epistaxis no se observó diferencia significativa en la frecuentación entre los días de la semana y los meses del año ( $>00,5)$.

Para comparar de forma adecuada las diferentes clases de epistaxis con distintas variables clínicas, como tipo de tratamiento y posibles asociaciones causales, comparamos las dos muestras

Tabla 2. Datos comparativos epistaxis anterior - epistaxis posterior.

\begin{tabular}{|c|c|c|c|c|c|}
\hline & & & Frecuencias absolutas & $\begin{array}{l}\text { Frecuencias según } \\
\text { tipo epistaxis }\end{array}$ & $\begin{array}{c}\text { Significación } \\
\text { estadística }\end{array}$ \\
\hline \multirow{4}{*}{ Sexo } & \multirow{2}{*}{ EA } & Hombre & 1.332 & $64,53 \%$ & \multirow{4}{*}{ P: 0,310} \\
\hline & & Mujer & 732 & $35,46 \%$ & \\
\hline & \multirow{2}{*}{$\mathrm{EP}$} & Hombre & 52 & $70,27 \%$ & \\
\hline & & Mujer & 22 & $29,73 \%$ & \\
\hline \multirow{8}{*}{ Edad (años) } & \multirow{4}{*}{ EA } & $<15$ & 245 & $11,87 \%$ & \multirow{8}{*}{ P: 0,015} \\
\hline & & $15-21$ & 108 & $5,23 \%$ & \\
\hline & & $22-50$ & 365 & $17,68 \%$ & \\
\hline & & $>50$ & 1.346 & $65,21 \%$ & \\
\hline & \multirow{4}{*}{$\mathrm{EP}$} & $<15$ & 3 & $4,05 \%$ & \\
\hline & & $15-21$ & 1 & $1,35 \%$ & \\
\hline & & $22-50$ & 21 & $28,37 \%$ & \\
\hline & & $>50$ & 49 & $66,21 \%$ & \\
\hline \multirow{10}{*}{$\begin{array}{c}\text { Situación al } \\
\text { alta }\end{array}$} & \multirow{5}{*}{ EA } & Domicilio & 2.052 & $99,41 \%$ & \multirow{10}{*}{$\mathrm{P}<0,001$} \\
\hline & & Hospitalización & 0 & $0 \%$ & \\
\hline & & Alta voluntaria & 1 & $0,04 \%$ & \\
\hline & & Fuga & 11 & $0,53 \%$ & \\
\hline & & Traslado & 0 & $0 \%$ & \\
\hline & \multirow{5}{*}{$\mathrm{EP}$} & Domicilio & 4 & $5,4 \%$ & \\
\hline & & Hospitalización & 64 & $86,48 \%$ & \\
\hline & & Alta voluntaria & 0 & $0 \%$ & \\
\hline & & Fuga & 0 & $0 \%$ & \\
\hline & & Traslado & 6 & $8,1 \%$ & \\
\hline \multirow{6}{*}{$\begin{array}{l}\text { Unidad de } \\
\text { gestión }\end{array}$} & \multirow{3}{*}{ EA } & Urgencias & 1.141 & $55,28 \%$ & \multirow{6}{*}{ P: 0,003} \\
\hline & & ORL & 902 & $43,70 \%$ & \\
\hline & & Pediatría & 21 & $1,01 \%$ & \\
\hline & \multirow{3}{*}{$\mathrm{EP}$} & Urgencias & 25 & $33,78 \%$ & \\
\hline & & ORL & 47 & $63,51 \%$ & \\
\hline & & Pediatría & 2 & $2,70 \%$ & \\
\hline
\end{tabular}




\section{ESTUDIO RETROSPECTIVO SOBRE LA INCIDENCIA Y TRATAMIENTO DE EPISTAXIS EN UN ÁREA DE SALUD EN EL PERÍODO 2011 A 2017 \\ HELLÍN-VALIENTE E ET AL}

Tabla 3. Datos comparativos de la muestra recogida (epistaxis anterior y epistaxis posterior).

\begin{tabular}{|c|c|c|c|c|c|}
\hline & & & $\mathrm{n}$ & $\%$ & $\mathrm{p}$ \\
\hline \multirow{4}{*}{ Sexo } & \multirow{2}{*}{ EA } & Varón & 43 & $48 \%$ & \multirow{4}{*}{0,001} \\
\hline & & Mujer & 47 & $52 \%$ & \\
\hline & \multirow{2}{*}{$\mathrm{EP}$} & Varón & 39 & $78 \%$ & \\
\hline & & Mujer & 11 & $22 \%$ & \\
\hline \multirow{8}{*}{ Edad } & \multirow{4}{*}{ EA } & $<15$ años & 17 & $18,88 \%$ & \multirow{8}{*}{0,001} \\
\hline & & $15-21$ años & 5 & $5,55 \%$ & \\
\hline & & $22-50$ años & 11 & $12,22 \%$ & \\
\hline & & $>50$ años & 57 & $63,33 \%$ & \\
\hline & \multirow{4}{*}{$\mathrm{EP}$} & $<15$ años & 0 & $0 \%$ & \\
\hline & & $15-21$ años & 0 & $0 \%$ & \\
\hline & & $22-50$ años & 14 & $28 \%$ & \\
\hline & & $>50$ años & 36 & $72 \%$ & \\
\hline \multirow{2}{*}{\multicolumn{2}{|c|}{ HTA }} & EA & 45 & $50 \%$ & \multirow{2}{*}{0,650} \\
\hline & & EP & 27 & $54 \%$ & \\
\hline \multirow{2}{*}{\multicolumn{2}{|c|}{$\mathrm{DM}$}} & EA & 15 & $16 \%$ & \multirow{2}{*}{0,622} \\
\hline & & EP & 10 & $20 \%$ & \\
\hline \multirow{2}{*}{\multicolumn{2}{|c|}{ DLP }} & EA & 21 & $23 \%$ & \multirow{2}{*}{0,387} \\
\hline & & $\mathrm{EP}$ & 15 & $30 \%$ & \\
\hline \multirow{2}{*}{\multicolumn{2}{|c|}{ FA }} & EA & 9 & $10 \%$ & \multirow{2}{*}{0,175} \\
\hline & & EP & 9 & $18 \%$ & \\
\hline \multirow{2}{*}{\multicolumn{2}{|c|}{ Cardiopatía isquémica }} & EA & 6 & $6,6 \%$ & \multirow{2}{*}{0,308} \\
\hline & & EP & 5 & $10 \%$ & \\
\hline \multirow{2}{*}{\multicolumn{2}{|c|}{$\begin{array}{l}\text { Antiagregación / } \\
\text { Anticoagulación }\end{array}$}} & EA & 16 & $17,7 \%$ & \multirow{2}{*}{0,102} \\
\hline & & EP & 15 & $30 \%$ & \\
\hline \multirow{9}{*}{ Tratamiento } & \multirow{4}{*}{ EA } & TA & 39 & $43 \%$ & \multirow{9}{*}{$<0,001$} \\
\hline & & $\mathrm{TA}+\mathrm{C}$ & 20 & $22 \%$ & \\
\hline & & TA+ingreso & 1 & $1 \%$ & \\
\hline & & No tto. & 30 & $33 \%$ & \\
\hline & \multirow{5}{*}{ EP } & SDB & 19 & $38 \%$ & \\
\hline & & $\mathrm{TP}$ & 11 & $22 \%$ & \\
\hline & & $\mathrm{SDB}+\mathrm{TP}$ & 6 & $12 \%$ & \\
\hline & & $\mathrm{TP}+\mathrm{C}$ & 9 & $18 \%$ & \\
\hline & & $\mathrm{TP}+\mathrm{E}$ & 5 & $10 \%$ & \\
\hline \multirow{2}{*}{\multicolumn{2}{|c|}{ Traslado }} & EA & 0 & $0 \%$ & \multirow{2}{*}{$<0,001$} \\
\hline & & EP & 5 & $10 \%$ & \\
\hline & ución & EA & 0 & $0 \%$ & $<0 \Omega 01$ \\
\hline & usion & EP & 4 & $8 \%$ & $<0,001$ \\
\hline & & EA & 0 & $0 \%$ & $<0001$ \\
\hline & (gid & EP & 23 & $46 \%$ & $<0,001$ \\
\hline
\end{tabular}

EA: epistaxis anterior; EP: epistaxis posterior. TA: taponamiento anterior; TP: taponamiento posterior; C: cauterización; SDB: sonda de doble balón; E: embolización. 


\section{ESTUDIO RETROSPECTIVO SOBRE LA INCIDENCIA Y TRATAMIENTO DE EPISTAXIS EN UN ÁREA DE SALUD EN EL PERÍODO 2011 A 2017 \\ HELLÍN-VALIENTE E ET AL}

de pacientes de cada grupo de epistaxis elegidos de forma aleatoria con 90 epistaxis anteriores y 50 posteriores, con los resultados recogidos en laTabla 3.

Se comparó la presencia de factores asociados en cada tipo de epistaxis, como se muestra en la Figura 2. La hipertensión arterial (HTA) estaba presente en 45 pacientes con epistaxis anterior (50\%), y en 27 pacientes con epistaxis posterior (54\%), no observándose una diferencia significativa $(p>0,5)$. La diabetes mellitus (DM) estaba presente en 15 pacientes con epistaxis anterior (16\%), y en 10 pacientes con epistaxis posterior (20\%), sin relación estadísticamente significativa ( $p>0,5$ ). La dislipemia (DLP) estaba presente en 21 pacientes con epistaxis anterior (23\%), y en 15 pacientes con epistaxis posterior $(30 \%)(\mathrm{p}>0,1)$. La fibrilación auricular (FA) estaba presente en 9 pacientes con epistaxis anterior (10\%), y en 9 pacientes con epistaxis posterior $(18 \%)(\mathrm{p}>0,1)$. La cardiopatía isquémica estaba presente en 6 pacientes con epistaxis anterior $(6,6 \%)$, y en 5 pacientes con epistaxis posterior $(10 \%)(\mathrm{p}>0,1)$. La antiagregación o anticoagulación estaba presente en 16 pacientes con epistaxis anterior $(17,7 \%)$, y en 15 pacientes con epistaxis posterior (30\%) $(\mathrm{p}>0,1)$. No consta datos de pacientes con patología hematológica.

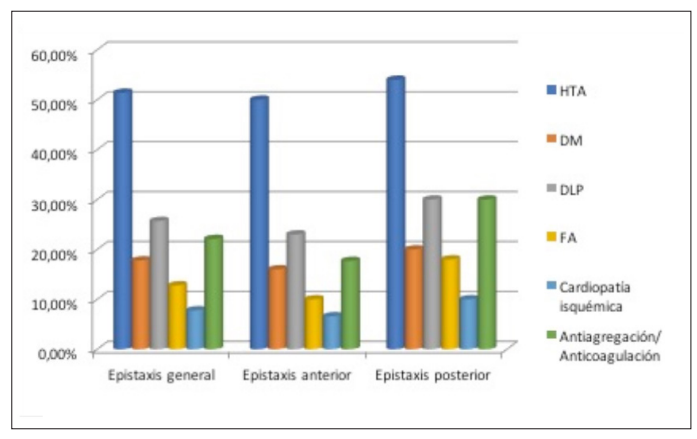

Figura 2. Frecuencia comorbilidades asociadas según tipo epistaxis.
No se observó diferencia entre el origen de la epistaxis y la lateralidad de la fosa nasal, teniendo la misma frecuencia la epistaxis derecha e izquierda.

En cuanto al rango de edad en la muestra recogida, como observamos en la Figura 3, comprobamos que en las epistaxis anteriores hubo 17 pacientes menores de 15 años, 5 pacientes entre 15 y 21 años, 11 pacientes entre 22 y 50 años, $y$ 57 pacientes mayores de 50 años. En las epistaxis posteriores, comprobamos que no hubo ningún paciente menor de 22 años, 14 pacientes entre $22 \mathrm{y}$ 50 años, y 36 pacientes mayores de 50 años. Hubo diferencia estadísticamente significativa (p: 0,001).

La edad media de las epistaxis anteriores fue de 54,80 años con una desviación típica de 25,58. En las posteriores, la edad media fue de 58,89 con una desviación típica de 20,29 ( $\mathrm{p}<0,05)$.

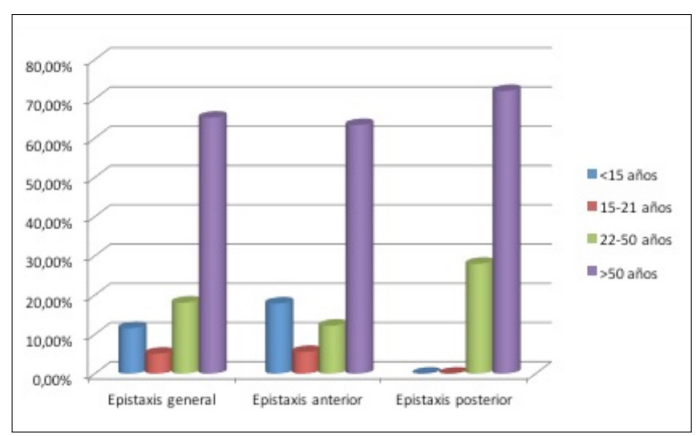

Figura 3. Frecuencia epistaxis según rango de edad.

La edad media de los pacientes con epistaxis $\mathrm{y}$ antiagregados o anticoagulados (74,67 años), es significativamente mayor que en los no antiagregados o anticoagulados (47,89 años) $(\mathrm{p}<0,001)$. La edad media de los pacientes con DM (69,72 años), es significativamente mayor que en los pacientes sin DM (50,75 años) ( $\mathrm{p}<0,001)$.

La edad media de los pacientes con HTA (69,13 años), es significativamente mayor que en los pacientes no hipertensos, con una edad media de 38,26 años $(\mathrm{p}<0,001)$. 


\section{ESTUDIO RETROSPECTIVO SOBRE LA INCIDENCIA Y TRATAMIENTO DE EPISTAXIS \\ EN UN ÁREA DE SALUD EN EL PERÍODO 2011 A 2017 \\ HELLÍN-VALIENTE E ET AL}

En cuanto al sexo, el $48 \%$ de las epistaxis anteriores eran varones, $\mathrm{y}$ en las posteriores fueron el $78 \%(\mathrm{p}<0,001)$.

Respecto al tipo de tratamiento recibido, en las epistaxis anteriores 20 pacientes $(22,2 \%)$ recibieron taponamiento anterior y cauterización, 1 paciente $(1,1 \%)$ precisó taponamiento anterior con ingreso, 39 pacientes $(43,3 \%)$ necesitaron taponamiento anterior, y 30 pacientes $(33,3 \%)$ no necesitaron tratamiento, siendo el sangrado autolimitado. En las epistaxis posteriores, 19 pacientes (38\%) recibieron sonda con doble balón, 11 pacientes (22\%) necesitaron taponamiento posterior, 6 pacientes (12\%) precisaron sonda y taponamiento posterior, 9 pacientes (18\%) precisaron taponamiento posterior y cauterización, y 5 pacientes $(10 \%)$ necesitaron taponamiento posterior y embolización ( $\mathrm{p}<0,001)$. Todos ellos fueron ingresados, ya sea en nuestro hospital o en el de referencia.

Estudiando las epistaxis en general, se observó que los tratamientos que más se realizaron para tratar la epistaxis fueron taponamiento anterior $(27,9 \%)$, taponamiento anterior y cauterización $(20,7 \%)$ o incluso sin necesidad de tratamiento por ser autolimitado $(21,4 \%)$. Los tratamientos menos realizados fueron taponamiento posterior y embolización $(3,6 \%)$ y sonda y taponamiento posterior $(4,3 \%)$. El tipo de taponamiento anterior más utilizado fue el de paquetes de Spongostan ${ }^{\circledR}$ (60\%), seguido de taponamientos con gasa (37\%), recurriendo al Merocel $^{\circledR}$ solo de forma ocasional.

En los pacientes con epistaxis posterior, fue necesario el traslado a otro centro en 5 pacientes (10\%), mientras que en las epistaxis anteriores no se realizó traslado en ningún paciente $(\mathrm{p}<0,001)$.

En los pacientes con epistaxis posterior, fue necesario tratamiento quirúrgico en 23 pacientes (46\%), mientras que no fue necesario en ningún paciente con epistaxis anterior $(p<0,001)$.

La transfusión sanguínea fue necesaria en 4 pacientes $(8 \%)$ con epistaxis posterior, y en ningún paciente con epistaxis anterior $(\mathrm{p}<0,001)$.
En cuanto a la media de los días de ingreso en los pacientes hospitalizados (solo en epistaxis posterior), es de 6,06 días, con una desviación típica de 6,159.

La duración media del taponamiento en la epistaxis posterior fue de 3,71 días, con una desviación típica de 2,245.

\section{DISCUSIÓN}

La epistaxis es un signo clínico y motivo de consulta frecuente en urgencias hospitalarias. Es la principal causa de sangrado nasosinusal suponiendo un 3,72\% de los pacientes atendidos en urgencias ORL, siendo la causa más frecuente de consulta por sintomatología ORL el catarro o infección de vías altas (19,7\%). Si solo tenemos en cuenta los pacientes atendidos por médicos especialistas de ORL el porcentaje se eleva al 10,32\%. En la literatura, se indica la epistaxis como motivo de urgencia más frecuente, oscilando entre el 13\% a $25 \%[4,5,15-17]$. Con menos frecuencia se menciona como patología muy frecuente, sin ser la primera causa $[18,19]$. Nosotros observamos una frecuencia inferior a la citada en la literatura. Esto puede ser debido a la inclusión en el estudio de todos aquellos pacientes con patologías originadas en region nasosinusal, faringe, laringe, oído, cara y cuello de forma general, y no solo las atendidas por el especialista.

Debido a que la mayoría de los pacientes con epistaxis no solicitan atención médica, su incidencia y prevalencia en la población general es difícil de calcular. Se ha observado en diferentes estudios una incidencia de entre 0,6 a 1,7 casos por 1.000 habitantes y año, y una prevalencia de hasta el $60 \%$ $[7,20]$. Nosotros hemos observado una incidencia de 1,754 casos por 1.000 habitantes y año de epistaxis general, acorde a lo obtenido en la literatura.

En cuanto a la clasificación de las epistaxis, encontramos en la literatura que la epistaxis anterior representa entre el $73-86 \%$ del total $[5,7,9$, $10,21]$. 


\section{ESTUDIO RETROSPECTIVO SOBRE LA INCIDENCIA Y TRATAMIENTO DE EPISTAXIS \\ EN UN ÁREA DE SALUD EN EL PERÍODO 2011 A 2017 \\ HELLÍN-VALIENTE E ET AL}

En nuestro estudio esta diferencia es más llamativa, encontrando epistaxis anterior en el $96,5 \%$ de los pacientes estudiados, y posterior en el 3,5\%. Es probable que esta diferencia puede ser incluso mayor, ya que una buena proporción de epistaxis anteriores son autolimitadas y los pacientes no solicitan atención médica, no pudiendo ser incluidas, por tanto, en estos estudios.

Respecto al sexo, hemos observado en nuestro estudio, un predominio de epistaxis en varones $(64,7 \%)$, con una ratio varón:mujer de 2:1. Estos datos son similares a los encontrados en la literatura, con un promedio de $66 \%$ varones y $33 \%$ mujeres $[4,7,9,10,17]$. Diferentes estudios sugieren que esta diferencia de prevalencia entre los sexos se deba en parte a la acción protectora de los estrógenos en la mujer [22, 23].

Al comparar el sexo en epistaxis anteriores y posteriores, la incidencia es mayor en varones en ambos tipos de epistaxis, pero esta diferencia es mucho mayor en las epistaxis posteriores, donde los varones representan el 78\% de nuestra muestra. Estos datos son similares a los consultados en la bibliografía [7], existiendo, al igual que en nuestro estudio, una diferencia estadísticamente significativa.

Respecto a la edad, observamos que la gran mayoría de nuestros pacientes fueron mayores de 50 años (65,2\%), con una edad media de 54,94 años, destacando un aumento de la frecuencia de la epistaxis a mayor edad. Algunos autores observan una distribución bimodal de la edad, con una mayor frecuencia en menores de 22 años y un segundo pico entre 61 y 80 años [7, 20].

Al comparar los pacientes con epistaxis anterior y posterior, observamos que los mayores de 50 años son el grupo mayoritario en ambos tipos. En las epistaxis anteriores los rangos de edad son más uniformes, pero predominan en los menores de 22 años (17,03\%), siendo posteriores solo el $5,4 \%$. De hecho, al comparar las edades medias en ambos grupos, vemos que en las anteriores la edad media es de 54,80 años, y en las posteriores es de 58,89 años. En la literatura, como nosotros, se observa que ambos tipos de epistaxis son más prevalentes en los mayores de 50 años [9]. En la literatura, en general, la epistaxis anterior tiene una mayor prevalencia en todas las edades, aunque las epistaxis posteriores prevalecen en los mayores de 80 años [7]. En cuanto a los rangos de edad en la muestra estudiada, obtenemos una diferencia estadísticamente significativa comparando ambos tipos de epistaxis (p: 0,001). Ningún paciente con epistaxis posteriores tenía menos de 22 años. Al igual que en la literatura, observamos el patrón bimodal en las epistaxis anteriores.

Como era de esperar, los pacientes con comorbilidad añadida (HTA, DM, antiagregación o anticoagulación) que presentan epistaxis tienen una edad media estadísticamente significativa mayor que los pacientes que no presentan estas patologías añadidas.

De todos los pacientes estudiados, el 96\% fueron dados de alta a domicilio, mientras que un 3,7\% necesitó ingreso hospitalario. Al comparar los grupos vemos que prácticamente todas las epistaxis anteriores fueron dadas de alta a domicilio $(99,4 \%)$, mientras que todas las posteriores fueron hospitalizadas en nuestro centro o trasladados e ingresados tras embolización a nuestro hospital de referencia. En la literatura se observa una mayor frecuencia de ingresos hospitalarios, entre el $6 \%$ a $8 \%[4,7,20]$, pero quizá justificado por una mayor frecuencia de epistaxis posteriores, puesto que son estas las que requieren ingreso. Comelli et al. [11], publican datos similares a los nuestros.

La mayoría de las anteriores fueron atendidos por urgencias al ser menos graves y la mayoría de las posteriores, más complejas, fueron atendidas por especialistas ORL.

En nuestro estudio observamos una importante variación estacional en la incidencia de epistaxis, siendo más prevalente en invierno y primavera, datos similares a los obtenidos en la bibliografía $[4,7,11,16,20,21,24]$. Esto puede justificarse por los cambios de temperatura y humedad y por 


\section{ESTUDIO RETROSPECTIVO SOBRE LA INCIDENCIA Y TRATAMIENTO DE EPISTAXIS \\ EN UN ÁREA DE SALUD EN EL PERÍODO 2011 A 2017 \\ HELLÍN-VALIENTE E ET AL}

la mayor incidencia de infecciones de vías altas y rinitis alérgica que se producen en estas estaciones. Sin embargo, se ha citado que el número de epistaxis diaria no se relaciona con la temperatura diaria [11].

Los días de atención más frecuente son los lunes $(18,3 \%)$ y los domingos el de menor frecuentación $(8,9 \%)$. Estos datos coinciden con Hijano et al. [18], y difieren de otras publicaciones que citan una mayor frecuentación los fines de semana [14]. Probablemente las epistaxis menos graves no demandan atención los días festivos.

No existe diferencia significativa en cuanto a la fosa de origen de la hemorragia siendo exactamente la misma en ambas fosas, hecho no mencionado en la literatura.

Se ha relacionado la epistaxis con diferentes factores de riesgo sistémicos, como son la HTA, DM, DLP, FA, cardiopatía isquémica y la antiagregación o anticoagulación normalmente por patología cardiovascular o cerebrales. Nosotros los hemos estudiado en la muestra recogida, y observamos que todos ellos son más frecuentes en las epistaxis posteriores, que también son más graves, por lo que se podría establecer una relación, aunque esta asociación no es estadísticamente significativa en ninguno de los grupos. Hay diferentes estudios que muestran estos factores de riesgo más prevalentes en las epistaxis $[4,9,10,21,25]$. Otros estudios, que relacionan exclusivamente la HTA con la epistaxis posterior, observan cifras más elevadas de TA en la epistaxis posterior con una alta significación estadística [26].

En una revisión sistemática realizada en 2013 [27], en la que se analizó la relación entre HTA y epistaxis, no se pudo establecer una relación causal entre el aumento de la TA y el episodio agudo de epistaxis, puesto que existen factores de confusión como el estrés o el fenómeno de la «bata blanca», por tanto, no se puede conocer si la HTA es una causa o consecuencia de la epistaxis. En un metanálisis en el que se estudia la asociación HTA y epistaxis se ha observado que el riesgo de epistaxis estaba aumentado de forma significativa en los pacientes con HTA [28].

En cuanto al tratamiento utilizado en la epistaxis en general, los más usados han sido el taponamiento anterior (27,9\%), taponamiento anterior y cauterización (20,7\%) y ausencia de tratamiento en la epistaxis autolimitada $(21,4 \%)$, mientras que el taponamiento posterior fue usado en el 8,6\% de los pacientes. En la mayoría de estudios se observa una mucha mayor frecuencia de taponamientos anteriores que de taponamientos posteriores [9, 19], esto se debe a que también hay una mayor frecuencia de epistaxis anteriores que posteriores. Algunos estudios indican que la cauterización fue realizada con mayor frecuencia que el taponamiento anterior [21]. Otros estudios señalan que la cauterización solo se realizó en alrededor del 3\% de los pacientes $[5,9,19]$.

Al comparar ambos grupos de epistaxis, vemos que en las anteriores, el tratamiento más realizado ha sido el taponamiento anterior $(43,3 \%)$, mientras que el $33,3 \%$ fueron autolimitadas, sin tratamiento médico, solo con compresión digital. Estos datos concuerdan con la literatura donde se establece el taponamiento anterior como el tratamiento más usado en las epistaxis anteriores [5]. Aunque cabe recordar que estos datos se refieren a pacientes que consultan en urgencias por epistaxis, por lo cual, asumimos que el tratamiento más frecuentemente realizado es la compresión digital, haciendo el sangrado autolimitado y no precisando así atención médica por parte del paciente. En las epistaxis posteriores, el tratamiento más realizado ha sido el taponamiento con sonda de doble balón (38\%), y el segundo en frecuencia el taponamiento posterior (22\%). Retuert R. et als [25], señalan un $42 \%$ de taponamientos posteriores, precisando cirugía en el $57 \%$. El $46 \%$ de los pacientes con epistaxis posterior precisaron tratamiento quirúrgico (embolización, cauterización, o taponmiento posterior bajo anestesia general), y ninguno en las epistaxis anteriores. En la literatura se publica una incidencia mayor de tratamiento quirúrgico, alrededor 


\section{ESTUDIO RETROSPECTIVO SOBRE LA INCIDENCIA Y TRATAMIENTO DE EPISTAXIS \\ EN UN ÁREA DE SALUD EN EL PERÍODO 2011 A 2017 \\ HELLÍN-VALIENTE E ET AL}

del 57\% [25]. El 8\% de nuestros pacientes precisó transfusión sanguínea, cifra similar a lo observado en la bibliografía, que cita un 10\% [19].

En cuanto a los pacientes ingresados, hemos observado una estancia media hospitalaria de 6,06 días. Esto concuerda con los datos obtenidos en la bibliografía donde se observa estancia media de entre 4 a 7 días $[4,21,25]$.

El $10 \%$ de la muestra de nuestros pacientes precisó traslado a otro centro por precisar embolización selectiva de la arteria esfenopalatina por el servicio de radiología intervencionista del Hospital Universitario Virgen de la Arrixaca. No encontramos en la literatura datos respecto a la frecuencia de este tratamiento.

El tiempo medio de permanencia del taponamiento posterior es de 3,71 días, datos coincidentes con lo obtenido en la literatura [25].

\section{CONCLUSIONES}

La epistaxis es un motivo de consulta frecuente en la consulta urgente de ORL. La incidencia relativa aumenta con edad, siendo excepcionales las epistaxis posteriores en la población infantil

Son más frecuentes en varones, principalmente las epistaxis posteriores. La incidencia de epistaxis aumenta en primavera e invierno. Son factores asociados la HTA, DM, DLP, FA, antiagregación o anticoagulación. La mayoría son de origen anterior, se atienden por médicos de urgencias, son autolimitadas o precisan taponamiento anterior, sin ingreso hospitalario. Las epistaxis posteriores son generalmente atendidas por médicos ORL, y precisan taponamiento posterior $\mathrm{y}$, si no cede, embolización de la arteria esfenopalatina.

\section{CONFLICTO DE INTERESES}

Los autores declaran no tener ningún conflicto de intereses.

Este estudio fue presentado como Trabajo de Fin de Grado realizado por la autora, en la Facultad de Medicina de la Universidad Católica de Murcia (UCAM), el día 5 de Junio de 2018.

\section{BIBLIOGRAFÍA}

1. Arruti G, Echeverría X, Medina JJ, Mozota O, Munilla M. Tratamiento de la epistaxis. Estudio de 235 casos. Anales ORL Iber Amer. 2008; 16(5):52741.

2. Villwock JA, Jones K. Recent trends in epistaxis management in the United States: 20082010. JAMA Otolaryngol Head Neck Surg 2013; 139:1279.

3. Harrison A. Approach to the adult with epistaxis. In: Uptodate. Jul 26, 2017. http://www.uptodate. com/

4. Monjas-Cánovas I, Hernández-García I, MauriBarberá J, Sanz-Romero B, Gras-Albert JR. Epidemiología de las epistaxis ingresadas en un hospital de tercer nivel. Acta Otorrinolaringol Esp. 2010;61(1):41-7.

5. Abraham ZS, Chugulu S, Liyombo E, Massawe ER, Ntunaguzi D. Prevalence of Epistaxis among Patients Receiving Otorhinolaryngology Services at Muhimbili National Hospital and Muhimbili Orthopedic Institute, Dar es Salaam, Tanzania. Medical Journal of Zambia. 2017; 44 (3): 184-92

6. Paparella M, Shumrick D. Otorrinolaringología. Tomo III. Cabeza y cuello. Buenos Aires, Editorial Médica Panamericana, 1982 pp 1979-93.

7. Vaamonde P, Martín C, Cajade J, Mínguez I, Lechuga R, Frade C et al. Datos epidemiológicos sobre la epistaxis: estudio hospitalario y revisión de la literatura. Acta Otorrinolaringológica Gallega. 2003; 4:1-8.

8. Schlosser RJ. Clinical practice. Epistaxis. N Engl J Med 2009; 360:784.

9. Sampigethya S, Cherian E, Pratap D, Mani I, Bhat VS. A clinical study of epistaxis. International Journal of Otorhinolaryngology and Head and Neck Surgery. 2018; 4 (2):555-8.

10. Bhaumik NC, Sukla B, Das B, Dey D, Nandi TK. Pattern of Epistaxis of Patients Attending in A Tertiary Care Hospital of Tripura, Northeastern Region of India. IOSR-JDMS. 2016; 15 (5):42-4. 


\section{ESTUDIO RETROSPECTIVO SOBRE LA INCIDENCIA Y TRATAMIENTO DE EPISTAXIS \\ EN UN ÁREA DE SALUD EN EL PERÍODO 2011 A 2017 \\ HELLÍN-VALIENTE E ET AL}

11. Comelli I, Vincenti V, Benatti M, Macri G, Cervellin G, Lippi G et al. Influence of air temperature variations on incidence of epistaxis. Am J Rhinol Allergy. 2015;29(6):175-81.

12. Soto-Galindo GA, Treviño JL. Epistaxis diagnosis and treatment update: A review. Ann Otolaryngol Rhinol. 2017;4(4):1176.

13. Alvi A, Joyner-Triplett N. Acute epistaxis. How to spot the source and stop the flow. Postgrad Med 1996;99:83.

14. Boettiger O. Otorrinolaringología. II Parte: Nariz, cavidades perinasales, boca, faringe, laringe. Santiago de Chile, Saval, 2000. pp 45-56.

15. Pino V, Trinidad G, González A, Pardo G, Pantoja $\mathrm{CG}$, Marcos $\mathrm{M}$ et al. Consideraciones sobre las urgencias ORL. Análisis de 30.000 pacientes atendidos en 10 años. Acta Otorrinolaringol Esp. 2005;56(5):198-201.

16. Walker T, Macfarlane T, McGarry G. The epidemiology and chronobiology of epistaxis: an investigation of Scottish hospital admissions 1995-2004. Clin Otolaryngol. 2007;32(5):361-5.

17. Raj A, Wadhwa V, Jain A. Epidemiological Profile of ENT Emergencies: Our Experience. Indian J Otolaryngol Head Neck Surg. 2018. Disponible en: https://www.springermedizin.de/epidemiological-profile-of-ent-emergencies-our-experience/15474590. [Citado el 12 de julio de 2018].

18. Hijano R, Hernández A, Martínez-Arias À, Homs I, Navarrete ML. Estudio epidemiológico de las urgencias en un hospital de tercer nivel. Acta Otorrinolaringol Esp. 2009;60(1):32-37.

19. Moran N, Das D. Epistaxis-Incidence, Etiology, and Management: A Hospitalbased Study. Clinical Rhinology An International Journal. 2016;9(1):18-20.

20. Pallin DJ, Chng YM, McKay MP, Emond JA, Pelletier AJ, Camargo CA Jr. Epidemiology of epistaxis in US emergency departments, 1992 to 2001. Ann Emerg Med. 2005;46:77-81.

21. Dangol B, Shrestha N, Yadav D, Devkota I, Nepal A, Bhandari S. Epistaxis at Patan Hospital: A Retrospective Review and an Audit. Nepalese Journal of ENT Head Neck Surg. 2014; 5 (2); 26-30.

22. Daniell HW. Estrogen prevention of recurrent epistaxis. Arch Otolaryngol Head Neck Surg $1995 ; 121: 354$

23. Fishpool SJ, Tomkinson A. Patterns of hospital admission with epistaxis for 26,725 patients over an 18-year period in Wales, UK. Ann R Coll Surg Engl 2012; 94:559.

24. Purkey M, Seeskin Z, Chandra R. Seasonal variation and predictors of epistaxis. The Laryngoscope. 2014;124(9):2028-33.

25. Retuert D, Fuentealba D, Bretón A, Ricci L, Nazar R, Naser A. Manejo epistaxis posterior en hospital clinico Universidad de Chile 2013 - 2016. Rev Otorrinolaringol. Cir. Cabeza Cuello. 2017; 77 (4). Disponible en: https:// scielo.conicyt.cl/scielo.php?script=sci_arttext\& pid=S0718-48162017000400389. [Citado el 12 de julio de 2018].

26. Para DM, Poenaru M, Marin AH, Doros C, Stefanescu H, Balica NC. Posterior Epistaxis and Hypertension: Our ENT Department Study. Laryng Rhin Otol. 2018;97(S02):S329-S330.

27. Kikidis D, Tsioufis K, Papanikolaou V, Zerva $\mathrm{K}$, Hantzakos A. Is epistaxis associated with arterial hypertension? A systematic review of the literature. Eur Arch Otorhinolaryngol. 2013;271(2):237-43.

28. Min H, Kang H, Choi G, Kim K. Association between Hypertension and Epistaxis: Systematic Review and Meta-analysis. Otolaryngol Head Neck Surg. 2017;157(6):921-7. 